\title{
Physiological Modeling for Analysis of Exhaled Breath ${ }^{1}$
}

\author{
Julian King, Helin Koc, Karl Unterkofler, Gerald Teschl, Susanne Teschl, \\ Pawel Mochalski, Hartmann Hinterhuber, and Anton Amann
}

\subsection{Introduction}

There is nothing more practical than a good theory. (David Hilber

Due to its broad scope and applicability, breath gas analysis holds great promise as a versatile framework for general bio-monitoring applications. As a biochemical probe, volatile organic compounds (VOCs) in exhaled breath are unique in the sense that they can provide both non-invasive and continuous information on the metabolic and physiological state of an individual. Apart from diagnostics and therapy control, this information might potentially be used for dynamic assessments of normal physiological function (e.g., by a stress test on a cycle ergometer, in an intra-operative setting, or in a sleep lab), pharmacodynamics (drug testing) or for quantifying body burden in response to environmental exposure (e.g., in occupational health) [3, 4, 2, 13, 46, 39. 44, 5]. Furthermore, as has been impressively demonstrated in the Nobel prize-winning work of Furchgott, Ignarro and Murad on the small inorganic molecule nitric oxide, trace gases can actively participate in the regulation of physiological events [23, 10]. This renders breath VOCs as an intriguing tool for examining more fundamental endogenous processes.

While sufficiently accurate and fast instrumental techniques are vital for fully exploiting this huge potential, also several methodological issues need to be addressed before breath gas analysis can become the "new blood test". Specifically, a crucial yet underestimated aspect of breath gas analysis concerns the blood-gas kinetics of the VOCs under scrutiny as well as their systemic distribution and physiological flow within the human body. Much can be learnt in this context from the cumbersome development of the nitric oxide breath test, which nowadays ranks among the most successful breath tests based on an endogenous compound [3]. This paradigmatic example shows that a purely explorative search for VOC biomarkers is not sufficient: in order to make breath tests operational, a thorough quantitative understanding of the underlying gas exchange mechanisms and their sensitivity with respect to physiological factors is

\footnotetext{
${ }^{1}$ To appear in: Volatile Biomarkers, Non-Invasive Diagnosis in Physiology and Medicine, A. Amann and D. Smith (eds), 1st Edition, Elsevier 2013 http://store.elsevier.com/Volatile-Biomarkers/isbn-9780444626134/

2also attributed to Immanuel Kant
} 
required. In particular, such knowledge can then contribute to the implementation of unified sampling protocols, similar to the ATS/ERS guidelines for determining exhaled lower respiratory tract nitric oxide and nasal nitric oxide [1].

Due to the current lack of definite sampling recommendations for other trace gases, end-tidal breath collection is usually adopted as best measurement practice. Accordingly, the first part of the exhalation - corresponding to the gas volume filling the anatomical dead space and hence not participating in gas exchange according to classical pulmonary inert gas elimination theory - is discarded and only the last exhalation segment (supposed to contain "alveolar air") is collected for analysis. In more sophisticated sampling systems, end-tidal extraction is triggered by virtue of simultaneously measured $\mathrm{CO}_{2}$ - and/or flow-data [20, 11]. While such setups are an important contribution to current standardization efforts in breath sampling, they generally give little insight into a series of major experimental questions:

- What is the quantitative relationship between breath VOC levels and their underlying endogenous (e.g., blood) VOC levels?

- Does the end-tidal VOC concentration level reflect the alveolar level?

- How does the variability of breath VOC concentrations relate to varying physiological conditions?

In this context, mathematical modeling and simulation can be employed to establish mechanistic descriptions of the gas exchange processes governing the VOC under scrutiny. Once such a model has been developed it becomes possible to understand the key relationships underlying the physiological behavior of the compound and to identify its defining parameters. Such a quantitative treatment provides a sound conceptual framework for experiment design as well as decision making and can aid substantially in preventing misinterpretations of empirical results.

Hence, in the following we shall try to answer the above questions by modeling the exhalation patterns of two highly abundant, endogenous trace gases found in human breath:

- isoprene, which has a low solubility in blood (as reflected by a small blood:gas partition coefficient $\lambda_{\text {b:air }} \approx 0.75$ ), is lipophilic and presumed to be correlated with cholesterol biosynthesis [50] and

- acetone, which is relatively much more soluble in blood $\left(\lambda_{\text {b:air }} \approx 340\right)$ and has been linked to fat catabolism.

Both isoprene and acetone can be viewed as prototypical examples for the analysis of two major classes of VOCs, even though they cannot cover the whole spectrum of different physico-chemical properties. Furthermore, these two compounds rank among the most notable VOCs studied in the context of breath gas analysis and thus represent natural choices for a more detailed assessment of observable trace gas behavior in exhaled breath.

\subsection{Real-time measurements: experimental basics}

The range of measurement techniques employed for breath gas analytical investigations is extremely diverse and each method comes with its specific strengths and weaknesses [4, 14]. Within the present setting, we will mainly focus on direct mass spectrometry, particularly proton transfer reaction mass spectrometry (PTR-MS) [36, 37, 


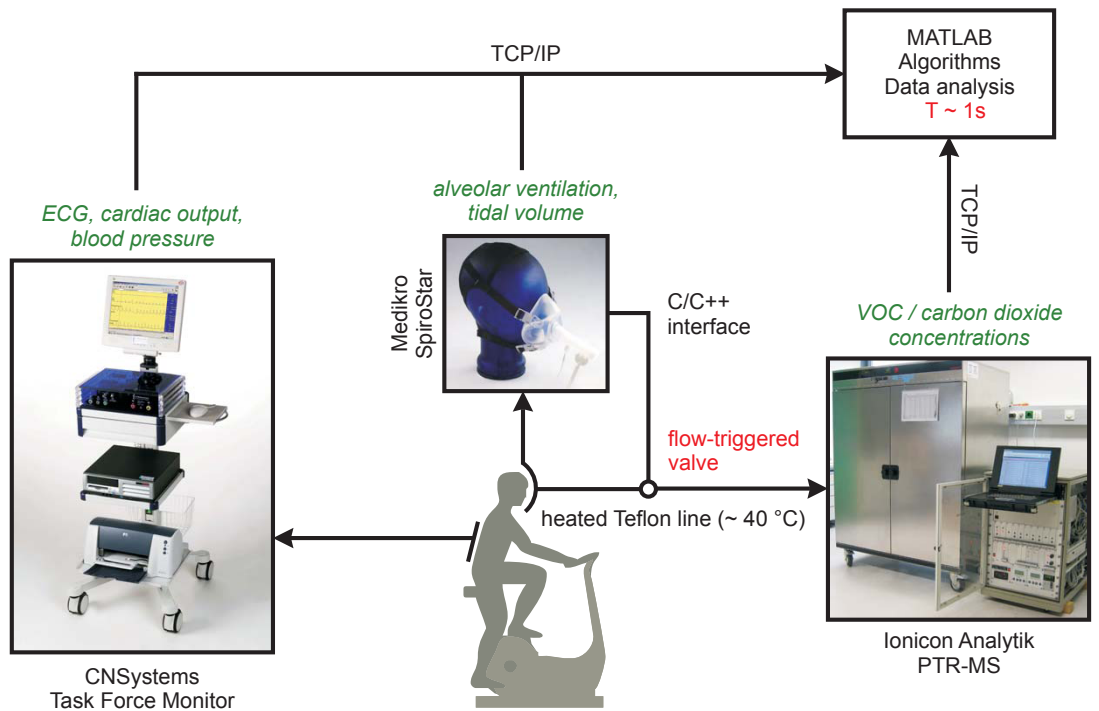

Figure 1.1: Experimental setup used for obtaining VOC profiles in conjunction with a number of physiological parameters, cf. [27]. Items in italic correspond to measurable variables. A selective analysis of predefined breath segments is achieved by flowtriggered sample extraction. More specifically, an automatic shutter valve installed along the gas sampling line guarantees that breath samples are drawn into the PTR-MS device exclusively during the end-tidal phase of each exhalation.

12, 19]. In brief, this technique has proven to be a sensitive method for the quantification of volatile molecular species on the basis of chemical ionization within a drift reactor tube. More specifically, it exploits the proton transfer reaction of precursor hydronium ions, $\mathrm{H}_{3} \mathrm{O}^{+}$, originating from an adjoint hollow cathode discharge, with molecules M, viz.,

$$
\mathrm{H}_{3} \mathrm{O}^{+}+\mathrm{M} \rightarrow \mathrm{MH}^{+}+\mathrm{H}_{2} \mathrm{O} \text {. }
$$

Specifically, this reaction process is selective to VOCs with proton affinities higher than water $(166.5 \mathrm{kcal} / \mathrm{mol})$, thereby avoiding the ionization of the bulk components of exhaled air, $\mathrm{N}_{2}, \mathrm{O}_{2}$ and $\mathrm{CO}_{2}$. Count rates of the nascent product ions $\mathrm{MH}^{+}$or fragments thereof appearing at specified mass-to-charge ratios $\mathrm{m} / z$ can subsequently be converted into absolute concentrations of the investigated compounds. Further details on quantification can be found in [48].

A major hallmark of PTR-MS is its real-time capability, allowing for concentration measurements of VOCs with breath-by-breath resolution (i.e., on a time-scale of less than $0.5 \mathrm{~s}$ ). The possibility of generating high sample rate data can be viewed as an essential requirement for relating short-term changes in breath VOC concentrations to quick physiological variations (e.g., in blood or ventilatory flow). An experimental setup combining real-time PTR-MS trace gas measurements with data streams reflecting hemodynamic and respiratory parameters is shown in Figure 1.1. For a detailed description the interested reader is referred to [27] (see also [29] for cross-validations by means of independent GC-MS data).

The above-mentioned instrumentation allows for the parallel evaluation of endtidal VOC concentration profiles and several decisive physiological factors during rest, exercise challenges on a cycle ergometer, and in a sleep laboratory setting []. Such 
synchronized data streams are shown in Figure 1.2 and represent an indispensable phenomenological basis pertinent to any quantitative modeling approach.

\subsection{Modeling}

In classical pulmonary inert gas elimination theory the relationship between the blood concentrations of VOCs and their respective concentrations in the gaseous phase is captured by the well-known Farhi equation [16]. It predicts a direct proportionality between the alveolar concentration $C_{\mathrm{A}}$ of a VOC (presumed to be accessible from end-tidal exhalation segments) and its concentration $C_{\mathrm{a}}$ and $C_{\overline{\mathrm{v}}}$ in arterial and mixed venous blood, respectively. Here, the associated proportionality factor depends on the substance-specific blood:gas partition coefficient $\lambda_{\text {b:air }}$ (describing the diffusion equilibrium at the alveolar-capillary interface according to Henry's law), alveolar ventilation $\dot{V}_{\mathrm{A}}$ (governing the transport of the compound through the respiratory tree), and cardiac output $\dot{Q}_{\mathrm{c}}$ (controlling the rate at which the VOC is delivered to the lungs), viz.,

$$
C_{\text {measured }}=C_{\mathrm{A}}=\lambda_{\mathrm{b}: \mathrm{air}} C_{\mathrm{a}}=\frac{C_{\overline{\mathrm{v}}}}{\lambda_{\mathrm{b}: \mathrm{air}}+\frac{\dot{V}_{\mathrm{A}}}{\dot{Q}_{\mathrm{c}}}} .
$$

An elementary but particularly useful tool for deriving such formulae is to employ the fundamental physical concept of mass balance coupled with a compartmental model design. Within a physiological framework, the compartmental approach to mass transfer traditionally consists in dividing the body into an ensemble of localized anatomic structures (like lung, liver, kidney, etc.) or roughly homogenous tissue volumes (lumping together tissue groups of a given type such as fat, muscle, viscera, bone, etc.) in which the investigated VOC can reasonably be postulated to behave uniformly. These compartments are subsequently interconnected via the arterial and venous network, yielding a simplified representation of the human organism that reflects known or hypothesized physiology and biochemistry [45, 35, 6, 18, 57]. A typical example of such a model is shown in Figure 1.3 .

Since their introduction in the 1930s [51, 52], compartment models have received widespread attention in the biomedical sciences. The reason for this popularity is that the model structures bear a direct correspondence with the underlying physiology, thereby maintaining a clear-cut interpretation of the involved parameters. Moreover, by writing down the associated mass balance relation s $^{3}$ the underlying model structure can directly be expressed as a set of ordinary differential equations (ODEs) of the form

$$
\dot{\mathbf{x}}(t)=\mathbf{g}(\mathbf{x}(t), \mathbf{u}(t), \boldsymbol{\vartheta}), \quad \mathbf{x}\left(t_{0}\right)=\mathbf{x}_{0} .
$$

These equations describe the evolution of a state vector $\mathbf{x}(t)$, belonging to an open set $X \subseteq \mathbb{R}^{n}$, within an observation interval $\left[t_{0}, t_{\max }\right]$. The right-hand side $\mathbf{g}$ usually is a continuously differentiable function with respect to its arguments and depends on a set of measured, possibly time-varying variables $\mathbf{u} \in U \subseteq \mathbb{R}^{q}$ that are postulated to be uniformly bounded over $\left[t_{0}, t_{\max }\right]$ as well as on a vector $\vartheta \in \Theta \subseteq \mathbb{R}^{p}$ lumping together a set of constant, unknown model parameters. The latter will have to be estimated from available process data (possibly together with some components of the

\footnotetext{
${ }^{3}$ stating that the rate at which the compartmental amount of a VOC changes per time unit equals the rate of mass transfer into the compartment less the rate of removal from the compartment
} 
Protocol A


Protocol B
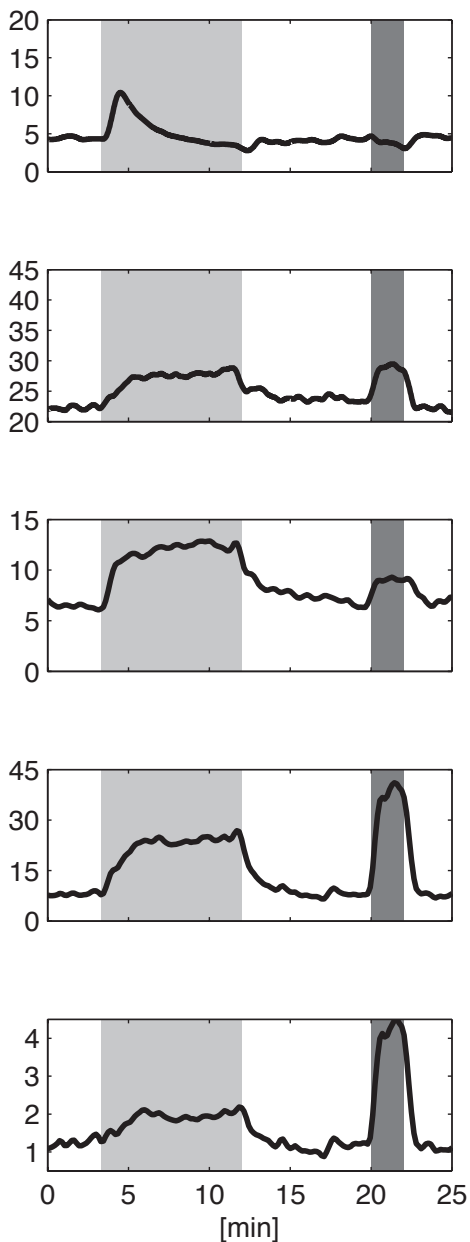

Figure 1.2: Typical smoothed profiles of end-tidal isoprene and acetone concentrations as well as hemodynamic and respiratory parameters in response to two distinct workload regimes. Segments shaded in light and dark grey indicate exercise and hyperventilation, respectively. Protocol A: rest (0-5 min), ergometer challenge at $75 \mathrm{~W}$ (5-20 $\mathrm{min})$, rest (20-32 $\mathrm{min})$, ergometer challenge at $75 \mathrm{~W}(32-47 \mathrm{~min})$, rest (47-50 $\mathrm{min})$, ergometer challenge at $75 \mathrm{~W}$ (50-55 min), rest (55-60 min). Protocol B: rest (0$3 \mathrm{~min}$ ), ergometer challenge at $75 \mathrm{~W}$ (3-12 min), rest (12-20 min), hyperventilation with increased tidal volume (20-22 $\mathrm{min})$, rest (22-25 min). Data correspond to one representative healthy male volunteer from the study cohort in [27]. 


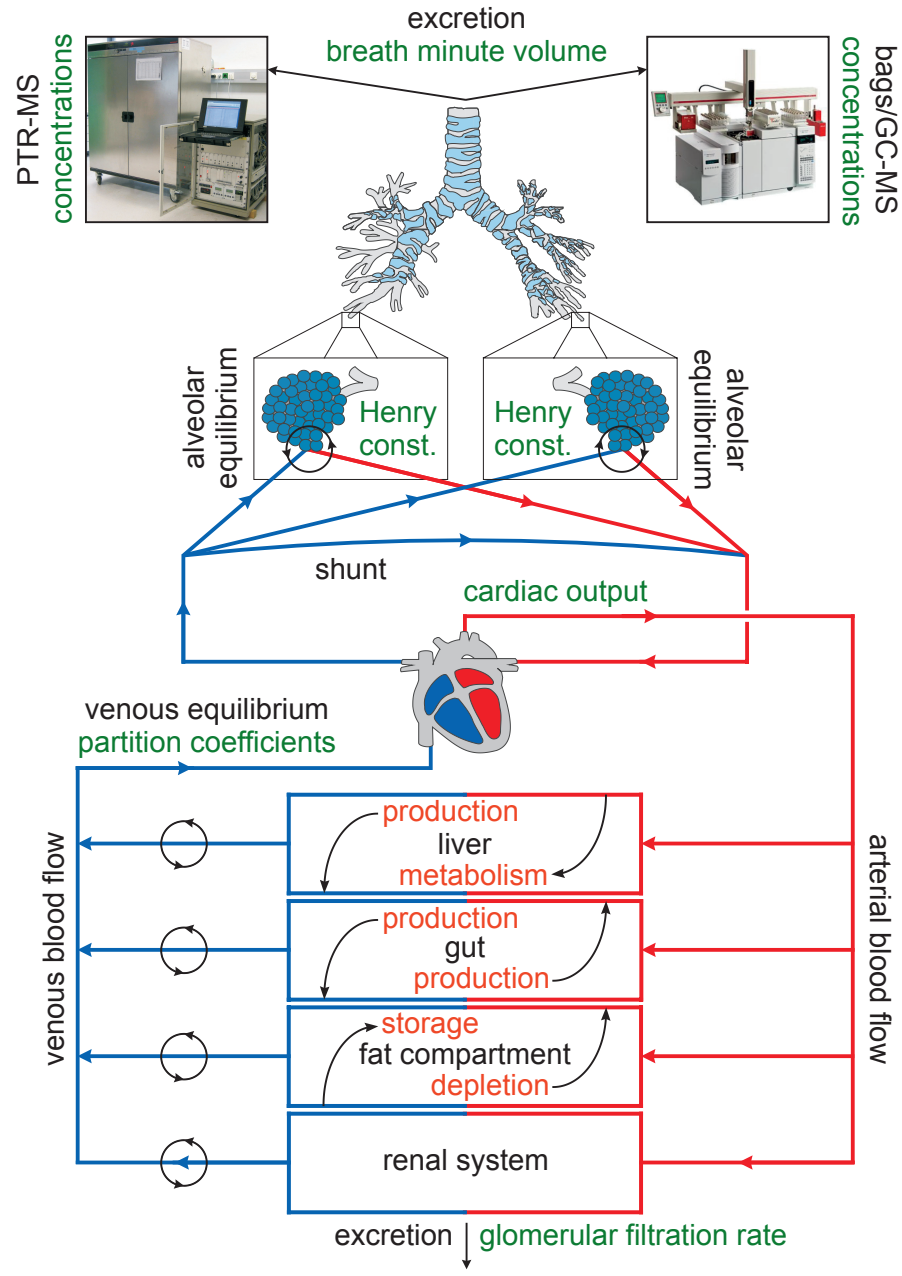

Figure 1.3: Physiologically based compartment model for the distribution of an endogenous volatile organic compound within the human body. The spectrum of model parameters can roughly be divided into fixed, measured, and unknown variables. The first class typically comprises quantities that have been determined a priori and are assumed to remain constant during the course of experimentation, such as approximate tissue volumes or partition coefficients. Information of this type has been compiled by several investigators, see, e.g., [45, 17] and references therein. Measured parameters include quantities that are accessible by virtue of the given experimental setup (blood flow, ventilation), while unknown parameters (e.g., production, metabolism, storage, and depletion rates) will have to be extracted by calibrating the model using available experimental data. 
initial conditions $\mathbf{x}_{0}$ ). In the present setting, $\mathbf{x}$ typically reflects a vector of molecular concentrations of the investigated trace gas within the tissue compartments introduced; u stands for external inputs (blood/respiratory flows, temperature, etc.) that can be modified by experimentation; $\boldsymbol{\vartheta}$ includes kinetic constants such as endogenous production or metabolization rates. Furthermore, information on the model dynamics can be collected via the (scalar) measurement equation

$$
y(t)=h(\mathbf{x}(t), \boldsymbol{\vartheta})
$$

where $h$ is some function defining the observable model output (i.e., the measurable breath concentration). Specific examples for the framework introduced above can be found in Sections 1.3.1 and 1.3.2

It is instructive to note that the Farhi equation represents a simple form of such a compartment model, involving only one gas exchange unit (the lungs) and assuming steady state conditions throughout the measurement (i.e., the rate of gas build-up/depletion in the lungs is zero). It is therefore not surprising that this formulation might be inadequate for explaining non-steady state behavior, e.g., in response to relatively quick physiological transitions (for instance during exercise). Indeed, from the data in Figure 1.2 the profiles of isoprene and acetone appear to considerably depart from the trend predicted by Equation (1.1). More specifically, at the onset of exercise the ventilation-perfusion ratio $\dot{V}_{\mathrm{A}} / \dot{Q}_{\mathrm{c}}$ increases drastically and hence, other factors being equal, a more or less pronounced drop in the respective breath concentrations could be expected 4

As we shall illustrate in the following, in the case of isoprene this discrepancy can be explained by reference to a peripheral (extrahepatic) production of the compound, which is realized by complementing the basic Farhi model with two systemic compartments affecting the time evolution of the mixed-venous concentration $C_{\overline{\mathrm{v}}}$ according to their fractional perfusion. For acetone, a mechanistic description of the profiles in Figure 1.2 can be achieved by taking into account pre- and post alveolar gas exchange. This amounts to dividing the pulmonary compartment into two parts, the alveolar tract and the conducting airways.

\subsubsection{Isoprene}

\section{Background}

Due to its volatility and low affinity for water and blood, isoprene exchange occurs mainly in the alveolar region. Physical activity causes marked changes in exhaled breath isoprene concentrations of humans [28, 24, 56]. Extensive real-time measurements during moderate workload ergometer challenges have been conducted by Karl et al. [24] and King et al. [28], showing an exercise-induced increase of breath isoprene concentrations by a factor of $\approx 2-3$ in mixed exhaled breath [24], and by a factor of $\approx 3-4$ in end-tidal breath [28]. Typical profiles are shown in Figure 1.2. While Karl et al. have explained this behavior by reference to a delay in the rise of ventilation with respect to the rise in perfusion at the start of exercise (i.e., by assuming a temporary drop in the average pulmonary ventilation-perfusion ratio), recent evidence points towards a substance-specific wash-out from an isoprene buffer tissue within the human body.

\footnotetext{
${ }^{4}$ Such a drop can in fact be observed for some endogenous breath VOCs, e.g., butane (cf. Figure 2 in ref. [29])
} 


\section{Some insightful experiments}

As can be deduced from Figure 1.2, exercise bouts at constant workload interrupted by breaks of variable duration lead to markedly different heights of the characteristic breath isoprene concentration peak at the onset of pedaling, despite an almost identical behavior of cardiac output and alveolar ventilation throughout all workload segments [28]. Additional information can be distilled from these profiles by performing one-legged ergometer experiments at moderate workloads [25], which appear to favor the hypothesis of a peripheral tissue source affecting breath isoprene output. More specifically, a switch of the working leg after a short break following an ergometer exercise segment at 50 Watts results in an immediate recovery of the initial peak height, whereas continuing the exercise with the same leg leads to a wash-out effect similar to the two-legged case.

Experiments carried out by stimulating muscle groups with distinct masses provide some hints with respect to the tissues involved in this behavior [32]. Breath isoprene concentration profiles during repeated arm-crank exercises with intermediate pauses qualitatively resemble the trends observed during ergometer challenges. However, compared to two-legged ergometer exercise, arm-crank exercise induces much lower peak isoprene concentrations, despite similar time courses of the ventilation-perfusion ratio within both exercise regimes. From these experiments it might hence be concluded that a major part of isoprene variability during exercise phases can be ascribed to an increased fractional perfusion of the working locomotor muscles, eventually leading to higher isoprene concentrations in mixed venous blood at the onset of physical activity. This rationale is in accordance with the predominant physiological role of working muscles during exercise. Collectively, the skeletal muscles constitute up to $40-45 \%$ of body weight, which is more than any other single organ. At rest, about 10$15 \%$ of cardiac output is distributed to skeletal muscle, while during strenuous exercise the latter may receive more than $80 \%$ of the total blood flow. This underlines the importance of skeletal muscle as one of the predominant factors in systemic hemodynamics [40].

\section{A physiological model for breath isoprene dynamics}

In the light of the experiments outlined above the mathematical model of isoprene distribution to be presented here adopts the assumption of a peripheral source of isoprene in the body. Following the methodology in Section 1.3 , the lung is modeled by one single alveolar unit and the systemic part consists of two homogenous compartments: a richly perfused tissue compartment (including intestines, liver, brain, connective muscles and skin) and a peripheral tissue compartment. The model structure is sketched in Figure 1.4 For the associated compartmental mass balance equations as well as model validation and estimation we refer to the original publication [26]. In order to correlate the model output to experimental outcomes, it is postulated that the measurable breath isoprene concentration corresponds to the alveolar level $C_{\mathrm{A}}$.

While production and metabolic elimination of isoprene occurs in both systemic compartments, peripheral tissue takes the role of an isoprene buffer and is postulated to contain the working muscle groups, which receive a disproportionally high fraction of the systemic blood flow during exercise. According to the simulation in Figure 1.5 at rest the peripheral compartment is characterized by high isoprene concentrations resulting from extrahepatic production at a constant rate. However, due to the relatively small fractional blood flow for these tissues, the mixed venous concentrations 


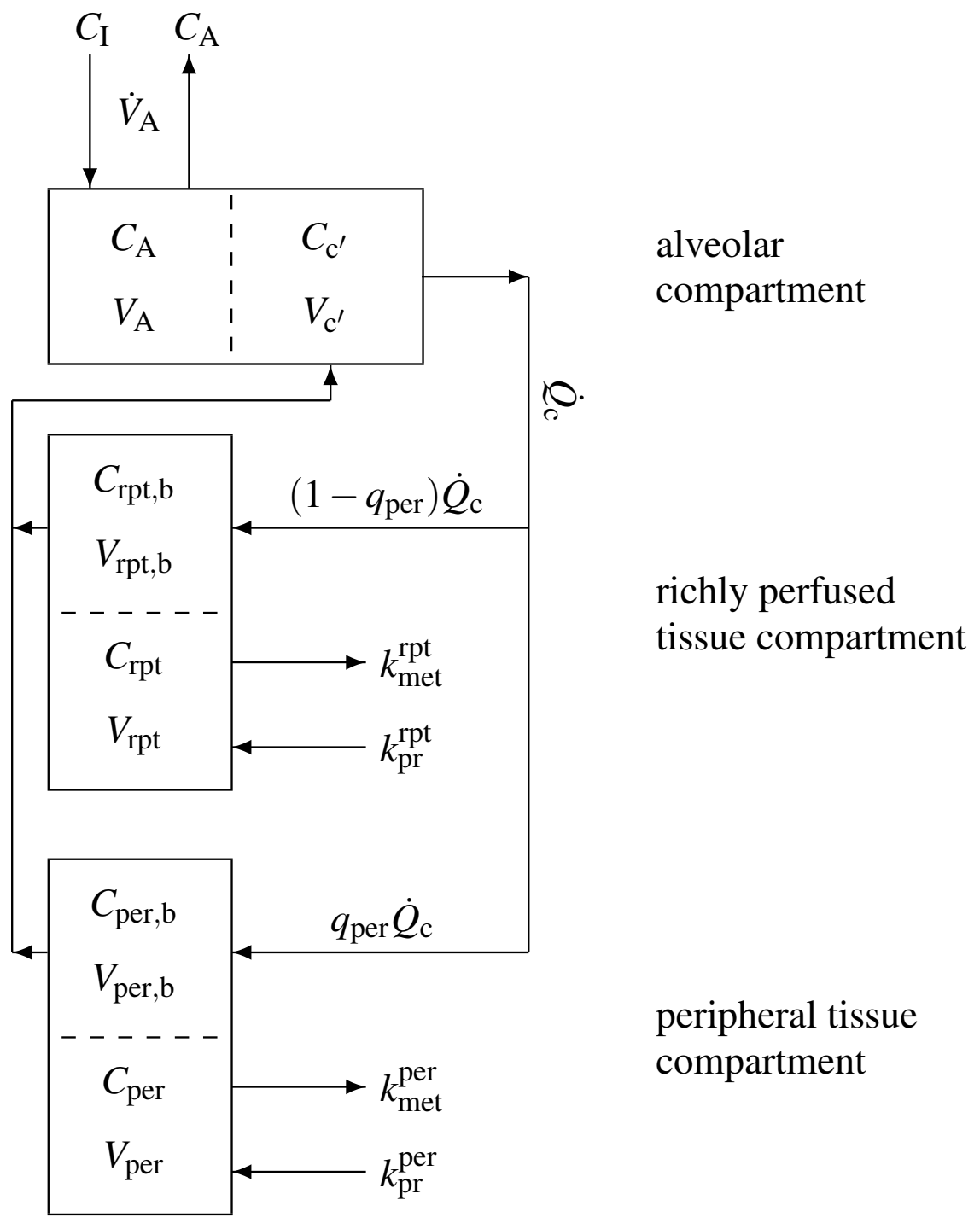

Figure 1.4: Schematic representation of the model structure used for describing the dynamics of isoprene concentrations $\left(C_{*}\right)$ in various parts of the human body. The latter is divided into three distinct functional units (with volumes $V_{*}$ ): alveolar/end-capillary compartment (gas exchange), richly perfused tissue (metabolism and production) and peripheral tissue (storage, metabolism and production). Fractional blood flow to the periphery is denoted by $q_{\mathrm{per}}$, while $k_{\mathrm{pr}}^{*}$ and $k_{\text {met }}^{*}$ represent constant production and (linear) metabolism rates, respectively. Dashed boundaries indicate a diffusion equilibrium. Subscripts denote as follows: I-inhaled; A-alveolar; $\mathrm{c}^{\prime}$-end-capillary; rptrichly perfused tissue; per-peripheral tissue; b-blood. 

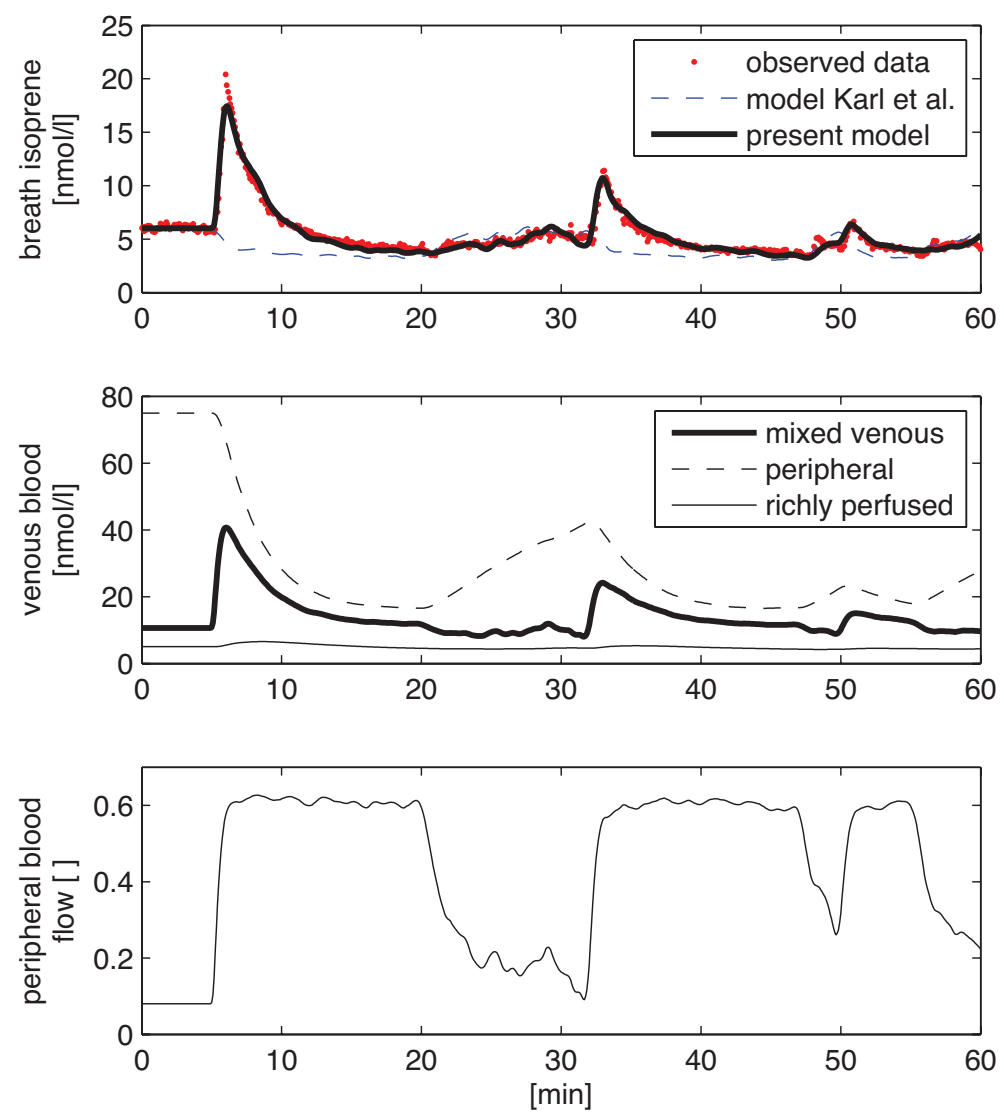

Figure 1.5: First panel: model response fitted to the isoprene breath data in Figure 1.2 Protocol A. Second panel: predicted concentrations in mixed venous blood and venous blood returning from the peripheral and richly perfused tissue groups. Third panel: predicted profile of fractional blood flow $q_{\text {per }}$ to peripheral tissue.

are mainly governed by the lower isoprene content of the venous blood returning from the richly perfused tissue group. At the start of exercise, the fractional perfusion $q_{\text {per }}$ in the periphery increases and consequently the mixed venous concentrations become dominated by peripheral venous return. The isoprene concentration peak visible in breath can thus be interpreted as a direct consequence of the associated increase in mixed venous blood concentrations.

\section{Practical implications}

Dynamic isoprene breath profiles corroborate the view that the breath output of certain VOCs can quickly vary with the physiological status of an individual. While a high sensitivity with respect to hemodynamics, respiration, food intake, etc. may impose strict regulations on the breath sampling protocol for such compounds, it can also provide further insights into their metabolic and physiological flow within the human 
body. In particular, for VOCs stemming from a specific endogenous (tissue) source, a change in the respective breath output should be observable if that source is activated, stimulated, or depressed. Using an analogous line of argument, exercise scenarios on an ergometer support the idea that some peripheral (muscle) tissue may play a role in isoprene synthesis. While this contrasts previous work postulating a purely hepatic origin of isoprene [15, 50], the above modeling study provides interesting new perspectives on the age dependence of isoprene output [49, 47, 34] as well as its reduction under statin therapy [24, 50]. Furthermore, it opens up the possibility for a mechanistic interpretation of future experimental findings and challenges new hypotheses.

\subsubsection{Acetone}

\section{Background}

In contrast to isoprene, acetone is both highly blood and water soluble. Hydrophilic compounds such as acetone have been demonstrated to interact with the water-like mucus membrane lining the conductive airways, an effect which has become known as wash-in/washout behavior. Extensive details can be found, e.g., in [54, 55, 7, 8]. Briefly, the term wash-in/wash-out refers to the fact that while fresh air is being inhaled, it becomes enriched with hydrophilic VOCs stored in the airway surface walls of the peripheral bronchial tract, thus leading to a decrease of the respective pressure/tension gradient between the gas phase and capillary blood in the alveolar space. This causes an effective reduction of the driving force for gas exchange in the alveoli and minimizes the unloading of the capillary VOC level. Correspondingly, during exhalation the aforementioned diffusion process is reversed, with a certain amount of hydrophilic VOCs being stripped from the air stream and redepositing onto the previously depleted mucus layer. As a phenomenological consequence, exhaled breath concentrations of such VOCs tend to be diminished on their way up from the deeper respiratory tract to the airway opening. The resulting discrepancies between the "true" alveolar and measured breath concentrations can be substantial (even if breath samples are drawn in a strictly standardized manner, e.g., by employing $\mathrm{CO}_{2}$-controlled sampling) and will depend on a variety of factors such as airway temperature profiles, airway perfusion, and volumetric flow.

\section{Some insightful experiments}

As in the case of isoprene, end-tidal acetone breath profiles during moderate ergometer challenges contradict the qualitative behavior anticipated from classical pulmonary inert gas elimination theory. From Figure 1.2, end-tidal acetone levels in response to exercise generally resemble the profile of ventilatory flow, showing abrupt increases and decreases in the range of $10-40 \%$ at the onsets and ends of the individual workload periods, respectively [28]. Similarly, during hyperventilation breath acetone levels tend to increase, whereas a (slight) decrease might be expected from Equation (1.1) (due to a drastic rise of the ventilation-perfusion ratio). Further evidence for the inadequacy of classical pulmonary inert gas elimination theory in describing the blood-breath relationships of hydrophilic compounds can be derived from isothermal rebreathing experiments [30]. The heuristic intention leading to isothermal rebreathing is to create an experimental situation diminishing the influence of the wash-in/washout mechanisms discussed above. This can be accomplished by "closing the respiratory loop", i.e., by continuous re-inspiration and re-exhalation of a fixed mass of air from a rebreathing 
receptacle (e.g., a Tedlar bag), causing the air stream to equilibrate with the mucosa linings over the entire respiratory cycle [22, 43, 42]. Additionally, warming the rebreathing volume to body temperature will ensure a similar solubility of these VOCs in both regions of the pulmonary tract, alveoli and airways. End-tidal breath profiles of highly water soluble VOCs such as acetone and methanol may increase by a factor of up to 1.5 during isothermal rebreathing [30], until approaching a plateau value that corresponds to the underlying alveolar concentration. Again, this behavior sharply contrasts with the formalism underlying Equation (1.1).

\section{A physiological model for breath acetone dynamics}

The experimental findings described above strongly suggest that airway gas exchange should be taken into account as a major determinant affecting the breath levels of hydrophilic VOCs. From a modeling viewpoint, this can be achieved by dividing the pulmonary tract into two separate compartments, an airway (bronchial) space and the alveolar space. The former comprises both a gas phase and a mucus membrane, which is assumed to have the physical properties of water and acts as a reservoir for acetone [33, 41]. Part of the acetone dissolved in this layer is transferred to the bronchial circulation, whereby a major fraction of the associated venous drainage is postulated to join the pulmonary veins via the post-capillary anastomoses [38]. The alveolar region itself is again represented by one single homogeneous alveolar unit. Similar to Section 1.3.1 the systemic part of the model consists of two functional units: a liver compartment, where acetone is endogenously produced and metabolized, as well as a passive tissue compartment representing an effective storage volume. The latter basically lumps together tissue groups with similar blood:tissue partition coefficient $\lambda_{\text {b:tis }} \approx 1.38$, such as richly perfused tissue, muscles and skin [9, 41]. A schematic representation of the model structure is presented in Figure 1.6. For the associated compartmental mass balance equations we again refer to the original publication [31]. In order to correlate the model output to experimental outcomes, it is postulated that the measurable breath acetone concentration corresponds to the bronchial level $C_{\text {bro }}$.

A direct implication of the wash-in/wash-out mechanisms discussed at the beginning of this section is that during free tidal breathing at rest the amount of hydrophilic VOCs reaching the alveolar region with each inhalation will be approximately equal to the amount extracted with each exhalation. In other words, under these circumstances the bronchio-alveolar net exchange of such compounds over one breath cycle will be close to zero and a major part of the overall pulmonary gas exchange (e.g., up to $96 \%$ as in the case of acetone [9]) can be ascribed to the conducting airways. As a consequence, a concentration gradient develops along the respiratory tree, with higher levels of the respective VOC found in the proximal (alveolar) parts. Increasing ventilatory flow rates and volume minimizes equilibration times with the more distal mucus layers and hence counteracts the saturation and depletion of the air stream during inhalation and exhalation, respectively. Consequently, the location of gas exchange is continuously shifted towards the alveolar region and the contribution of the latter becomes more pronounced. In particular, the end-exhaled (measured) breath concentration approaches values closer to the alveolar level.

This rationale can be incorporated into the model by including a diffusion term of the form $D\left(C_{\mathrm{bro}}-C_{\mathrm{A}}\right)$ into the underlying mass balance equations. This expression reflects the above-mentioned net gas exchange between the alveolar and the bronchial tract over a complete breath cycle (i.e., the conductance parameter $D$ is close to zero during free tidal breathing at rest and increases with ventilatory flow). 


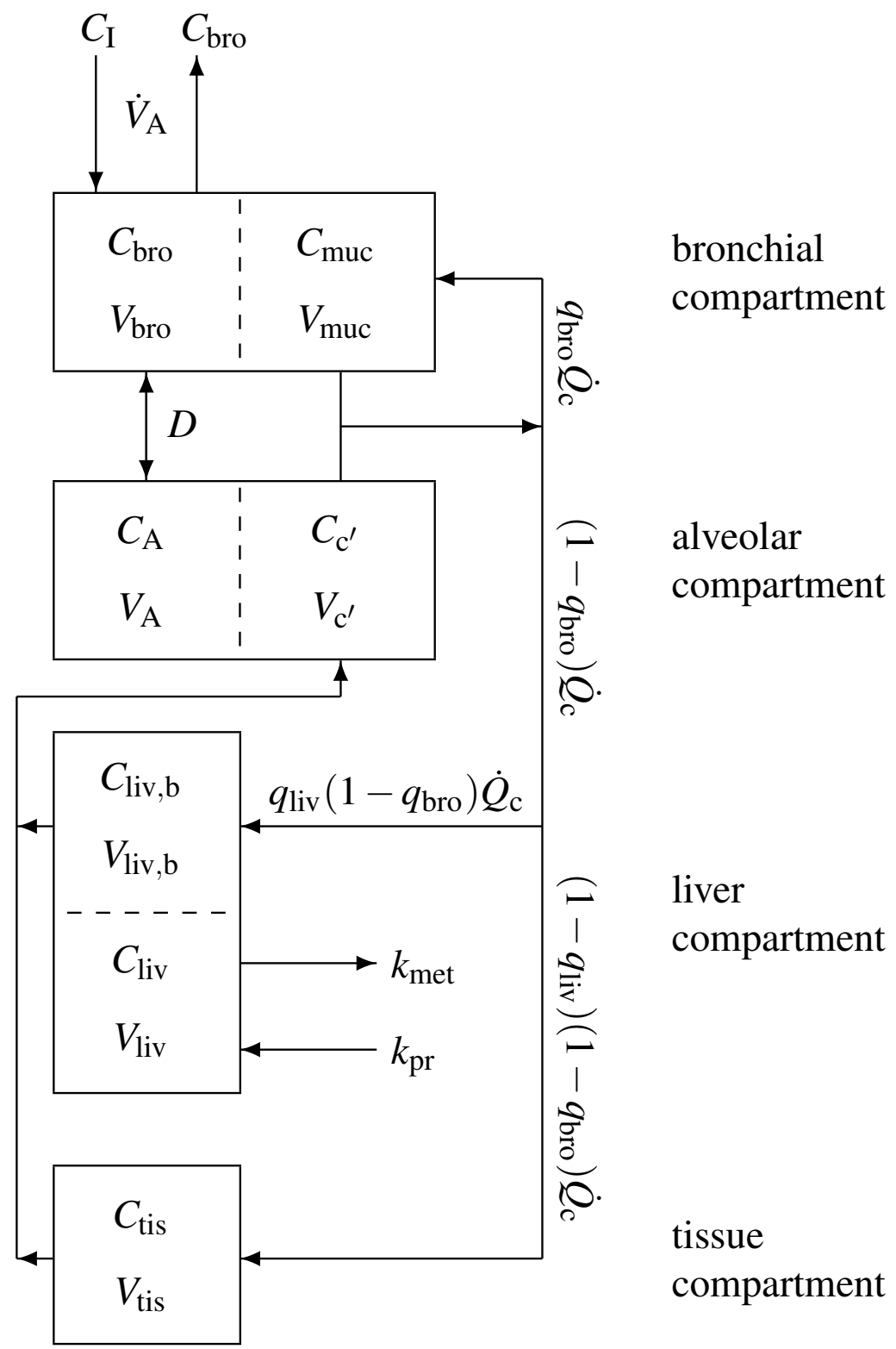

Figure 1.6: Schematic representation of the model structure used for describing the dynamics of acetone concentrations $\left(C_{*}\right)$ in various parts of the human body. The latter is divided into four distinct functional units (with volumes $V_{*}$ ): bronchial/mucosal compartment (gas exchange), alveolar/end-capillary compartment (gas exchange), liver (metabolism and production) and tissue (storage). Fractional blood flows are denoted by $q_{*}$, while $k_{\mathrm{pr}}^{*}$ and $k_{\text {met }}^{*}$ represent constant production and (linear) metabolism rates, respectively. Dashed boundaries indicate a diffusion equilibrium. Subscripts denote as follows: I-inhaled; bro-bronchial; muc-mucosal; A-alveolar; $c^{\prime}$-end-capillary; livliver; tis-tissue; b-blood. 
The relationships outlined in the previous paragraphs are summarized in Figure 1.7 . showing a calibrated version of the model with the observable model output (i.e., the bronchial concentration) fitted to the experimental data in Figure 1.2 Protocol B. It is interesting to note that the alveolar acetone concentration stays relatively constant throughout the entire experiment, i.e., the short-term variability observable in breath acetone concentrations can almost exclusively be attributed to airway gas exchange. Similarly, only minute changes in the underlying blood and tissue concentrations are expected to occur.

For the sake of completeness, it should be mentioned that another important factor affecting the gas exchange of hydrophilic VOCs is airway temperature. In particular, changes in the latter will affect the solubility of acetone and similar compounds in the mucus surface of the respiratory tree [54,22]. For example, the local solubility of acetone in the mucus layer increases from $\lambda_{\text {muc:air }}\left(37^{\circ} \mathrm{C}\right) \approx 392$ in the lower respiratory tract to $\lambda_{\text {muc:air }}\left(32^{\circ} \mathrm{C}\right) \approx 498$ at the mouth, thereby accentuating the wash-out of air stream acetone during the exhalation phase, cf. Section 1.3.2 Warming the airways to body core temperature can alleviate this effect by ensuring a uniform solubility distribution throughout the airways. This is consistent with an increase of the measured breath concentrations during isothermal rebreathing experiments [43, 42, 30]. For further details on how to incorporate explicit temperature dependences into the mechanistic model presented above, the reader is referred to reference [31]. This paper also further reports on the validation of the model, e.g., during exposure scenarios.

\section{Practical implications}

The major conclusion that can be drawn from quantitatively modeling exhalation dynamics of hydrophilic VOCs is that for this class of compounds the measured endexhaled breath concentrations and the underlying alveolar levels must not be taken as identical. For perspective, the experimental evidence outlined above indicates that for acetone during free tidal breathing at rest the associated values can differ by a factor of more than 1.5. In particular, this implies that multiplying end-tidal breath concentrations with the substance-specific in vitro blood:gas partition coefficient $\lambda_{\mathrm{b} \text { :air }}$ will generally result in an underestimation of the true arterial blood concentration of hydrophilic VOCs. In vivo, the (arterial) blood-breath ratio strongly depends on a variety of additional factors that can vary considerably among individuals, including airway temperature and bronchial blood flow (both exerting an inversely monotonous influence on the blood-breath ratio). Modeling can give a good mechanistic account of these relationships. In particular, it can be used to predict the impact of conditions/pathologies that are known to alter airway gas exchange (e.g., in asthma or bronchiectasis) and can provide hints for designing and evaluating sampling protocols aimed at improving the reliability and information content of breath measurements involving hydrophilic VOCs.

\subsection{Concluding remarks}

In summary, we believe that breath gas analysis would greatly profit from a more causal understanding of VOC kinetics in response to distinct physiological states. However, the development of quantitative formulations relating breath concentrations of trace gases to their underlying systemic levels clearly lags behind the enormous analytical progress in the field. This situation calls for the establishment of a proper modeling 



Figure 1.7: First panel: model response fitted to the acetone breath data in Figure 1.2 Protocol B. Second panel: corresponding concentrations in the bronchial, alveolar, liver, and tissue compartments. Third panel: measured respiratory parameters and the estimated time course of the conductance parameter $D$, reflecting the net gas exchange between the alveolar and the bronchial tract as described in the text. Note that $D$ is close to zero during free tidal breathing at rest (i.e., the measureable breath acetone levels are almost exclusively determined by the respective levels in the conducting airways) and varies in parallel with ventilatory flow. In particular, higher values of $D$ correspond to an increased contribution of the alveoli to total pulmonary gas exchange. 
framework, striving for validated mechanistic descriptions of VOC dynamics in different parts of the organism. As an outlook, from an operational point of view such modeling studies can

- help to identify potential confounding factors affecting the interpretation of breath test results and propose corrective measures.

- guide the ongoing standardization of sampling procedures and provide formal means for evaluating the information content of newly proposed experimental settings.

- enhance the fundamental understanding of the physiological role of VOCs, e.g., by relating phenomenological features of observable breath concentration profiles to specific distribution, transport, bio-transformation and excretion processes of these substances.

- stimulate biochemical investigations exploring the endogenous (e.g., cellular) sources of VOCs.

In particular, the last two points are directed towards further consolidating the potential role of breath gas analysis in general bio-monitoring applications.

\subsection{Acknowledgments}

J. King, H. Koc, and G. Teschl gratefully acknowledge support from the Austrian Science Fund (FWF) under Grant No.Y330. J. King, K. Unterkofler, and P. Mochalski gratefully acknowledge support from the Austrian Science Fund (FWF) under Grant No. P24736-B23. We greatly appreciate the generous support of the government of Vorarlberg, Austria. We appreciate funding from the Austrian Federal Ministry for Transport, Innovation and Technology (BMVIT/BMWA, project 836308, KIRAS). 


\section{Bibliography}

[1] ATS/ERS Recommendations for Standardized Procedures for the Online and Offline Measurement of Exhaled Lower Respiratory Nitric Oxide and Nasal Nitric Oxide, 2005, Am. J. Respir. Crit. Care Med. 171 (2005), 912-930.

[2] A. Amann, G. Poupart, S. Telser, M. Ledochowski, A. Schmid, and S. Mechtcheriakov, Applications of breath gas analysis in medicine, Int. J. Mass Spectrometry 239 (2004), 227-233.

[3] A. Amann and D. Smith (eds.), Breath analysis for clinical diagnosis and therapeutic monitoring, World Scientific, Singapore, 2005.

[4] A. Amann, P. Spanel, and D. Smith, Breath analysis: the approach towards clinical applications, Mini reviews in Medicinal Chemistry 7 (2007), 115-129.

[5] L. C. A. Amorim and Z. de L. Cardeal, Breath air analysis and its use as a biomarker in biological monitoring of occupational and environmental exposure to chemical agents, J. Chromatogr. B Analyt. Technol. Biomed. Life Sci. 853 (2007), 1-9.

[6] M. E. Andersen, Physiological modeling of organic compounds, Ann. Occup. Hyg. 35 (1991), 309-321.

[7] J. C. Anderson, A. L. Babb, and M. P. Hlastala, Modeling soluble gas exchange in the airways and alveoli, Ann. Biomed. Eng. 31 (2003), 1402-22.

[8] J. C. Anderson and M. P. Hlastala, Breath tests and airway gas exchange, Pulm. Pharmacol. Ther. 20 (2007), 112-117.

[9] J. C. Anderson, W. J. Lamm, and M. P. Hlastala, Measuring airway exchange of endogenous acetone using a single-exhalation breathing maneuver, J. Appl. Physiol. 100 (2006), 880-9.

[10] P. J. Barnes, Nitric oxide and airway disease, Ann. Med. 27 (1995), 389-393.

[11] T. Birken, J. Schubert, W. Miekisch, and G. Noldge-Schomburg, A novel visually $\mathrm{CO}_{2}$ controlled alveolar breath sampling technique Technol. Health Care 14 (2006), 499-506.

[12] Robert S. Blake, Paul S. Monks, and Andrew M. Ellis, Proton-transfer reaction mass spectrometry, Chemical Reviews 109 (2009), 861-896.
[13] B. Buszewski, M. Kesy, T. Ligor, and A. Amann, Human exhaled air analytics: biomarkers of diseases, Biomed. Chromatogr. 21 (2007), 553-566.

[14] W. Cao and Y. Duan, Current status of meth ods and techniques for breath analysis, Critical Reviews in Analytical Chemistry 37 (2007), 3 13.

[15] E S Deneris, R A Stein, and J F Mead, Acid catalyzed formation of isoprene from a mevalonate-derived product using a rat liver cytosolic fraction, J Biol Chem 260 (1985), 1382-5.

[16] L. E. Farhi, Elimination of inert gas by the lung, Respir. Physiol. 3 (1967), 1-11.

[17] V. Fiserova-Bergerova (ed.), Modeling of inhalation exposure to vapors: Uptake, distribution, and elimination, CRC Press, Boca Raton, 1983.

[18] L. E. Gerlowski and R. K. Jain, Physiologically based pharmacokinetic modeling: principles and applications, Journal of Pharmaceutical Sciences 72 (1983), 1103-1127.

[19] J. Herbig, M. Müller, S. Schallhart, T. Titzmann, M. Graus, and A. Hansel, On-line breath analysis with PTR-TOF, J. Breath Res. 3 (2009), 027004.

[20] J. Herbig, T. Titzmann, J. Beauchamp, and I. Kohl, Buffered end-tidal (BET) sampling a novel method for real-time breath-gas analysis, J. Breath Res. 2 (2008), 1-9.

[21] M P Hlastala and A J Berger, Physiology of respiration, 2nd ed., Oxford University Press, New York, 2001.

[22] A. W. Jones, Role of rebreathing in determination of the blood-breath ratio of expired ethanol, J. Appl. Physiol. 55 (1983), 12371241.

[23] D. E. Koshland Jr., The molecule of the year, Science 258 (1992), 1861

[24] T Karl, P Prazeller, D Mayr, A Jordan, $\mathrm{J}$ Rieder, R Fall, and W Lindinger, $\mathrm{Hu}$ man breath isoprene and its relation to blood cholesterol levels: new measurements and modeling, J Appl Physiol 91 (2001), 762-70. 
[25] J King, H Koc, K Unterkofler, P Mochalski, A Kupferthaler, G Teschl, S Teschl, H Hinterhuber, and A Amann, Physiological modeling of isoprene dynamics in exhaled breath, J Theor Biol 267 (2010), 626-37.

[26] J. King, H. Koc, K. Unterkofler, P. Mochalski, A. Kupferthaler, G. Teschl, S. Teschl, H. Hinterhuber, and A. Amann, Physiological modeling of isoprene dynamics in exhaled breath, $\mathrm{J}$ Theor. Biol. 267 (2010), 626-637.

[27] J. King, A. Kupferthaler, K. Unterkofler, H. Koc, S. Teschl, G. Teschl, W. Miekisch, J. Schubert, H. Hinterhuber, and A. Amann, Isoprene and acetone concentration profiles during exercise on an ergometer, J. Breath Res. 3 (2009), 027006 (16pp).

[28] J King, A Kupferthaler, K Unterkofler, H Koc, S Teschl, G Teschl, W Miekisch, J Schubert, $\mathrm{H}$ Hinterhuber, and A Amann, Isoprene and acetone concentration profiles during exercise on an ergometer, J Breath Res 3 (2009), 027006.

[29] J. King, P. Mochalski, A. Kupferthaler, K. Unterkofler, H. Koc, W. Filipiak, S. Teschl, H. Hinterhuber, and A. Amann, Dynamic profiles of volatile organic compounds in exhaled breath as determined by a coupled PTRMS/GC-MS study, Physiol. Meas. 31 (2010), 1169-1184.

[30] J. King, K. Unterkofler, A. Kupferthaler, G. Teschl, S. Teschl, H. Koc, H. Hinterhuber, and A. Amann, A modeling based evaluation of isothermal rebreathing for breath gas analysis of highly soluble volatile organic compounds, Preprint, 2010.

[31] J. King, K. Unterkofler, G. Teschl, S. Teschl, H. Koc, H. Hinterhuber, and A. Amann, A mathematical model for breath gas analysis of volatile organic compounds with special emphasis on acetone, J. Math. Biol. (2011), DOI 10.1007/s00285-010-0398-9.

[32] H. Koc, J. King, G. Teschl, K. Unterkofler, S. Teschl, P. Mochalski, H. Hinterhuber, and A. Amann, The role of mathematical modeling in voc analysis using isoprene as a prototypic example, J. Breath Res. (2011), accepted.

[33] S. Kumagai and I. Matsunaga, A lung model describing uptake of organic solvents and roles of mucosal blood flow and metabolism in the bronchioles, Inhal. Toxicol. 12 (2000), 491510 .

[34] I Kushch, B Arendacká, S Stolc, P Mochalski, W Filipiak, K Schwarz, L Schwentner, A Schmid, A Dzien, M Lechleitner, V Witkovský, W Miekisch, J Schubert, K Unterkofler, and A Amann, Breath isopreneaspects of normal physiology related to age, gender and cholesterol profile as determined in a proton transfer reaction mass spectrometry study, Clin Chem Lab Med 46 (2008), 1011-8.
[35] H. W. Leung, Development and utilization of physiologically based pharmacokinetic models for toxicological applications, J. Toxicol. Environ. Health 32 (1991), 247-267.

[36] W. Lindinger, A. Hansel, and A. Jordan, Online monitoring of volatile organic compounds at pptv levels by means of proton-transferreaction mass spectrometry (PTR-MS) medical applications, food control and environmental research, Int. J. Mass Spectrom. Ion Processes 173 (1998), 191-241.

[37] _ Proton-transfer-reaction mass spectrometry (PTR-MS): on-line monitoring of volatile organic compounds at pptv levels, Chem. Soc. Rev. 27 (1998), 347-354.

[38] A. B. Lumb, Nunn's applied respiratory physiology, 6th ed., Butterworth-Heinemann, Oxford, 2005.

[39] W. Miekisch and J. K. Schubert, From highly sophisticated analytical techniques to lifesaving diagnostics: Technical developments in breath analysis, Trends in Analytical Chemistry 25 (2006), 665-673.

[40] D E Mohrman and L J Heller, Cardiovascluar physiology, 6th ed., Lange Medical Books/McGraw-Hill, New York, 2006.

[41] A. K. Mörk and G. Johanson, A human physiological model describing acetone kinetics in blood and breath during various levels of physical exercise, Toxicol. Lett. 164 (2006), 6-15.

[42] M. E. O’Hara, S. O'Hehir, S. Green, and C. A. Mayhew, Development of a protocol to measure volatile organic compounds in human breath: a comparison of rebreathing and online single exhalations using proton transfer reaction mass spectrometry, Physiol. Meas. 29 (2008), 309-30.

[43] J. Ohlsson, D. D. Ralph, M. A. Mandelkorn, A. L. Babb, and M. P. Hlastala, Accurate measurement of blood alcohol concentration with isothermal rebreathing, J. Stud. Alcohol 51 (1990), 6-13.

[44] J. D. Pleil, Role of exhaled breath biomarkers in environmental health science, J. Toxicol. Environ. Health B Crit. Rev. 11 (2008), 613629.

[45] M. B. Reddy, R. S. H. Yang, H. J. Clewell III, and M. E. Andersen (eds.), Physiologically based pharmacokinetic modeling: science and applications, Wiley, Hoboken, 2005.

[46] J. Rieder, P. Lirk, C. Ebenbichler, G. Gruber, P. Prazeller, W. Lindinger, and A. Amann, Analysis of volatile organic compounds: possible applications in metabolic disorders and cancer screening, Wien. Klin. Wochenschr. 113 (2001), 181-5. 
[47] T H Risby, Volatile organic compounds as markers in normal and diseased states, Disease markers in exhaled breath: basic mechanisms and clinical applications, Series I: Life and Behavioural Sciences, vol. 346, IOS Press, Amsterdam, 2002, pp. 113-22.

[48] K. Schwarz, W. Filipiak, and A. Amann, Determining concentration patterns of volatile compounds in exhaled breath by PTR-MS, J. Breath Res. 3 (2009), 027002 (15pp).

[49] D Smith, P Spanel, B Enderby, W Lenney, $\mathrm{C}$ Turner, and S J Davies, Isoprene levels in the exhaled breath of 200 healthy pupils within the age range 7-18 years studied using SIFT-MS, J Breath Res 4 (2010), 017101.

[50] B. G. Stone, T. J. Besse, W. C. Duane, C. D. Evans, and E. G. DeMaster, Effect of regulating cholesterol biosynthesis on breath isoprene excretion in men, Lipids 28 (1993), 705-708.

[51] T. Teorell, Kinetics of distribution of substances administered to the body. I. The extravascular modes of administration, Arch. Int. Pharmacodyn. 57 (1937), 205-225.

[52] Kinetics of distribution of substances administered to the body. II. The intravascular modes of administration, Arch. Int. Pharmacodyn. 57 (1937), 226-240.

[53] S Timischl, A global model for the cardiovascular and respiratory system, $\mathrm{Ph} . \mathrm{D}$. thesis, Karl Franzens University of Graz, 1998.

[54] M. E. Tsu, A. L. Babb, D. D. Ralph, and M. P. Hlastala, Dynamics of heat, water, and soluble gas exchange in the human airways: 1. A model study, Ann. Biomed. Eng. 16 (1988), 547-571.

[55] M. E. Tsu, A. L. Babb, E. M. Sugiyama, and M. P. Hlastala, Dynamics of soluble gas exchange in the airways: 2. Effects of breathing conditions, Respir. Physiol. 83 (1991), 261276.

[56] C. Turner, P. Spanel, and D. Smith, A longitudinal study of breath isoprene in healthy volunteers using selected ion flow tube mass spectrometry (SIFT-MS), Physiol. Meas. 27 (2006), 13-22.

[57] P. D. Wagner, Handbook of Physiology, The Respiratory System, Gas Exchange, ch. Peripheral Inert-Gas Exchange, pp. 257-281, Oxford University Press, New York, 1987.

[58] P D Wagner, H A Saltzman, and J B West, Measurement of continuous distributions of ventilation-perfusion ratios: theory, J Appl Physiol 36 (1974), 588-98. 